\title{
Mediterranean diet and metabolic status in post-menopausal women living in a mediterranean area
}

\author{
Valerio Fiore $^{\mathrm{a}}$, Michele Capraro ${ }^{\mathrm{a}}$, Rosalia Ragusa ${ }^{\mathrm{b}}$, Justyna Godos $^{\mathrm{a}}$, Antonio Mistretta ${ }^{\mathrm{a}}$ \\ and Marina Marranzano ${ }^{\mathrm{a}, *}$ \\ a Department of Medical and Surgical Sciences and Advanced Technologies "G.F. Ingrassia”, University of \\ Catania, Catania, Italy \\ bAzienda Ospedaliera Universitaria Policlinico “Vittorio Emanuele”, Catania, Italy
}

\begin{abstract}
.
BACKGROUND: Limited data are available regarding the association between dietary habits and metabolic outcomes in post-menopausal women living in Italy.

OBJECTIVE: To investigate the relation between adherence to the Mediterranean diet and prevalence of overweight/obesity, type-2 diabetes, hypertension and dyslipidemias in a sample of post-menopausal women.

METHODS: Data on 499 women were extracted from a population-based representative sample collected in Sicily, southern Italy. High adherence to the Mediterranean diet was defined as being in the highest quartile of a score used to calculate the level of adherence in the entire cohort.

RESULTS: Only $15.8 \%(n=83)$ of the study sample was found to have high adherence to the Mediterranean diet; these individuals were less likely to be overweight [odds ratio $(\mathrm{OR})=0.52,95 \%$ confidence interval $(\mathrm{CI}): 0.31-0.86$ ] and obese $(\mathrm{OR}=0.38,95 \%$ CI: $0.18-0.78)$ as well as having dyslipidemias ( $\mathrm{OR}=0.50,95 \% \mathrm{CI}: 0.30-0.84)$. Among individual components of the Mediterranean diet adherence score used, obesity was inversely associated with fruit component, type-2 diabetes with cereals and olive oil, hypertension with fruit and dairy products, dyslipidemias with legumes and dairy products.

CONCLUSIONS: Higher adherence to the Mediterranean diet was associated with better metabolic status in post-menopausal women.
\end{abstract}

Keywords: Mediterranean diet, metabolic status, postmenopausal, diabetes

\section{Introduction}

Metabolic disorders contribute to the highest of proportion of risk factors for a healthy life, playing a role especially among older individuals [1]. Obesity is today a pandemic of gigantic proportion, especially in southern European regions [2]. Type 2 diabetes mellitus, elevated blood pressure and dyslipidemias play a crucial role on cardiovascular disease (CVD) risk [3]. Overall, the burden

\footnotetext{
*Corresponding author: Marina Marranzano, Department of Medical and Surgical Sciences and Advanced Technologies "G.F. Ingrassia”, University of Catania, Catania, Italy. Tel.: +39095 378 2180; E-mail:marranz@unict.it.
}

of metabolic disorders represents a public health issue for the general population due to their impact in determining global disability-adjusted life years $[4,5]$. However, some differences between men and women should be pointed out: CVD generally occurs in women ten years later than in men as in premenopausal women the clinical manifestations of CVD (acute myocardial infarction, stroke and sudden death) are relatively rare. On the other hand, in post-menopausal women, the occurrence and severity of CVD dramatically increase, nearly matching the respective risk observed in men. Besides hormonal differences, women may have specific risk factors that may affect their CVD risk, such as hysterectomy, ovariectomy, pre-menstrual syndrome, 
oral contraception and pregnancy complications such as systemic arterial hypertension, type-2 diabetes mellitus (DM), miscarriage and preterm birth [6-9]. However, menopause itself is characterized by a change in hormonal activity, which in turn alters metabolic homeostasis and may lead to metabolic imbalance [10].

In this context, the role of diet has been further investigated, as potential determinant of health in post-menopausal women $[11,12]$. There is convincing evidence draft from concordance between randomized controlled trials and meta-analyses of cohort studies showing a decreased risk of cardiovascular disease in individuals more adherent to a Mediterranean diet resembling the traditional dietary pattern compared with those who did not $[13,14]$. Despite with some differences between countries, some key points seem to be in common with most varieties of dietary patterns of individuals living in the Mediterranean area, such as the following: a high consumption of plant foods, including fruit and vegetables, but also legumes and nuts; a moderate consumption of dairy products and sea-food; a limited consumption of poultry and red meat, with avoidance of processed meats and sweets; use of extra-virgin olive oil as main source of dietary fats; and moderate consumption of alcohol, mainly red wine, generally during the meals [15]. These characteristics, all-together, provide a pattern of macroand micronutrients, as well as vitamins, phytochemicals and minerals that have been shown to be associated with prolonged lifespan in the general population [16]. Overall, the main benefits seem to affect cardio-metabolic risk factors resulting in a decreased risk of mortality rates among high adherent to this dietary pattern [17-19]. However, studies conducted in post-menopausal women, which may have different hormonal profile are scarce. The aim of this study was to evaluate the association between adherence to the Mediterranean diet and a pattern of metabolic conditions, including weight status, T2DM, hypertension and dyslipidemias in a sample of post-menopausal women living in Sicily, southern Italy.

\section{Methods}

\subsection{Study population}

The MEAL study is an observational study, which aim was to explore the relation between nutritional and lifestyle factors characterizing individuals liv- ing in the Mediterranean area. The detailed study protocol has been reported previously [20]. In brief, a random sample of general population, including men and women aged 18 years old and over registered in the records of local general practitioners (GPs), was selected in the urban area of Catania, southern Italy. The theoretical sample size was set at 1500 individuals to provide a specific relative precision of 5\% (Type I error, 0.05; Type II error, 0.10), with $70 \%$ participation rate. The sampling technique included stratification by municipality area, age, and sex of individuals, and randomization into subgroups, with randomly selected general practitioners being the sampling units, and individuals registered to them comprising the final sample units. The study did not include pregnant women. Women were invited to participate to the study while visiting the outpatient or called whether not reached out. Out of 2,405 individuals invited, the final sample size was 2,044 participants (response rate of $85 \%$ ).

\subsection{Data collection}

Data have been collected through measurements and structured with trained nurses or dieticians under the supervision of a medical doctor. Information was collected regarding demographic data, including sex, age, latest educational degree achieved, occupation (specifies the character of the most important employment during the year before the investigation) or last occupation before retirement, and marital status. Educational level was divided into the following categories: (i) low (primary/secondary), (ii) medium (high school), and (iii) high (university). Occupational level was divided as (i) unemployed, (ii) low (unskilled/unemployed workers), (iii) medium (partially skilled workers), and (iv) high (skilled workers). Additionally, information on smoking habits, alcohol drinking, and physical activity was recorded. Smoking status was divided into following categories: as (i) non-smoker, (ii) ex-smoker, and (iii) current smoker. Alcohol consumption was categorized as (i) none, (ii) moderate drinker (0.1-12 g/d) and (iii) regular drinker $(>12 \mathrm{~g} / \mathrm{d})$. Physical activity level was calculated using the International Physical Activity Questionnaires (IPAQ), which included a set of questionnaires (5 domains) investigating the time spent being physically active in the last 7 days: based on the IPAQ guidelines, final scores allows to categorized physical activity level as (i) low, (ii) moderate, and (iii) high. 


\subsection{Dietary assessment and adherence to the Mediterranean diet}

Food intake was investigated using a validated food frequency questionnaire (FFQ) [21, 22]. The Mediterranean diet adherence was assessed using an existing score from literature (MEDI-LITE score) [23]. In brief, a scoring system was built weighting all the median (or mean) values for the sample size of each study population and then calculating a mean value of all the weighted medians; thus, two standard deviations were used to determine three different categories of consumption for each food group. For foods, typical of the Mediterranean diet (fruit, vegetables, cereals, legumes and fish), 2 points were given to the highest category of consumption, 1 point for the middle category and 0 point for the lowest category. On the other hand, for food groups not typical of the Mediterranean diet (meat and meat products, dairy products), 2 points were given for the lowest category, 1 point for the middle category and 0 point for the highest category of consumption. Regarding alcohol intake, categories related to the alcohol unit ( 1 alcohol unit $=12 \mathrm{~g}$ of alcohol) were used by giving 2 points to the middle category (1-2 alcohol units/d), 1 point to the lowest category ( $>1$ alcohol unit/d) and 0 point to the highest category of consumption ( $>2$ alcohol units/d). The final adherence score included nine food groups (including olive oil) with a score ranging from 0 point (lowest adherence) to 18 points (highest adherence). Taking into consideration the fact that in our cohort we registered an average high adherence to the Mediterranean diet, we categorized the score as (i) low-to-moderate and (ii) high adherence to the Mediterranean diet, with a cut-off point of $\geq 15$.

\subsection{Anthropometric measurements and metabolic variables ascertainment}

Anthropometric measurements were collected using standardized methods [24]. Height was measured to the nearest $0.5 \mathrm{~cm}$ without shoes, with the back square against the wall, eyes looking straight ahead, with a right-angle triangle resting on the scalp and against the wall. Body mass index (BMI) was registered, and patients were divided into under/normal weight (BMI $<25 \mathrm{Kg} / \mathrm{m} 2$ ), overweight (BMI 25 to $29.9 \mathrm{Kg} / \mathrm{m} 2)$, and obese $(\mathrm{BMI}>30 \mathrm{Kg} / \mathrm{m} 2)$. Waist circumference (centimetres) was measured midway between the 12th rib and the iliac crest and hip circumference (centimetres) around the buttocks, at the level of the maximum extension. Waist-to-hip ratio was also calculated. At the end of the physical examination, arterial blood pressure was measured with subject in sitting position and at least 5 minutes at rest. Blood pressure measurements were performed three times at the right arm relaxed and well supported by a table, with an angle of $45^{\circ}$ from the trunk. A mean of the last two measurements was calculated and considered the analysis. Patients have been considered hypertensive when average systolic/diastolic blood pressure levels were greater or equal to $140 / 90 \mathrm{~mm}$ $\mathrm{Hg}$, taking anti-hypertensive medications, or being previously diagnosed of hypertension. In addition, patients were considered diabetic or dyslipidemic whether previously diagnosed of diabetes and hypercholesterolemia/hypertriglyceridemia, respectively.

\subsection{Statistical analysis}

Frequencies are expressed as numbers and percentages, while continuous variables are expressed as means and standard deviations. Differences between groups were calculated using Chi-square test for categorical variables, while Student's $t$-test or MannWhitney U-test were used for variables distributed normally and not normally, respectively. Multivariate logistic regression analyses were used in order to test association between background characteristics and the outcomes of interest, adjusted for potential confounding factors, including age, educational status, occupational status, marital status, smoking status, physical activity status, total energy intake ( $\mathrm{kcal} / \mathrm{d})$. All $P$ values were based on two-sided tests; significance level was considered as 5\%. SPSS 17 (SPSS Inc., Chicago, IL, USA) software was used in order to perform all statistical calculations.

\section{Results}

The main background characteristics of the post-menopausal women included in this study is presented in Table 1. The average age of the participants was 64 years, the majority had low educational and occupational status or were unemployed; half of the sample was married. Regarding cardiovascular risk factors, most of the women were non-smoker, had moderate physical activity level and were moderate alcohol drinkers. Concerning their metabolic status, most of them had hypertension, nearly half were overweight-obese and had dyslipidemias, while $10 \%$ had type- 2 diabetes. 
Table 1

Main background characteristics of the sample of post-menopausal women $(n=499)$

\begin{tabular}{lc}
\hline Characteristics & $n(\%)$ \\
\hline Age, mean (SD) & $64(11.8)$ \\
Educational status & \\
Low & $304(57.8)$ \\
Medium & $150(28.5)$ \\
High & $72(13.7)$ \\
Occupational status & \\
Unemployed & $379(72.1)$ \\
Low & $88(16.7)$ \\
Medium & 0 \\
High & 0 \\
Marital status & \\
Unmarried/single & $213(40.5)$ \\
Married/partner & $313(59.5)$ \\
Overweight-obese $(B M I>25)$ & $277(55.5)$ \\
Obesity (BMI $>30)$ & $120(24)$ \\
Hypertension & $356(71.3)$ \\
Type-2 diabetes & $54(10.8)$ \\
Dyslipidemia & $208(41.7)$ \\
Smoking status & \\
Non-smoker & $332(63.1)$ \\
Ex-smoker & $130(24.7)$ \\
Current smoker & $64(12.2)$ \\
Physical activity status & \\
Low & $144(27.4)$ \\
Moderate & $222(42.2)$ \\
High & $76(14.4)$ \\
Alcohol consumption & \\
None & $134(25.5)$ \\
Moderate drinker $(0.1-12 \mathrm{~g} / \mathrm{d})$ & $290(55.1)$ \\
Regular drinker $(>12 \mathrm{~g} / \mathrm{d})$ & $100(19.1)$ \\
\hline &
\end{tabular}

When calculating the adherence score for the Mediterranean dietary pattern and dividing it into quartiles, women in the highest quartile $(n=83)$ were tested for association with various metabolic outcomes compared to those with lower adherence to this dietary pattern, as shown in Table 2 . Findings from both the uni- and multivariate models were similar, resulting in higher adherence to the Mediterranean diet associated with less likelihood of overweight [odds ratio $(\mathrm{OR})=0.52,95 \%$ confidence interval (CI): 0.31-0.86], obesity $(\mathrm{OR}=0.38,95 \%$ CI: $0.18-0.78)$, and dyslipidemias (OR $=0.50,95 \%$ CI: 0.30-0.84). No further associations with other metabolic conditions were found. However, when the analyses were conducted for individual components of the Mediterranean diet adherence score used, several associations were found: obesity was inversely associated with fruit component, type- 2 diabetes with cereals and olive oil, hypertension with fruit and dairy products, dyslipidemias with legumes and dairy products (Table 3).

\section{Discussion}

In the present study, an inverse association between high adherence to the Mediterranean diet and prevalence of overweight/obesity and dyslipidemias was found in a sample of post-menopausal women living in Sicily, southern Italy. No association with type-2 diabetes and hypertension was revealed.

Studies conducted on post-menopausal women are not numerous and not necessarily reflect the findings referred to the whole general population. In some previous reports conducted on the general population living in Mediterranean islands, similar results regarding the association between high adherence to the Mediterranean diet and weight status have been found [25-27]. However, the relation between Mediterranean diet and metabolic health in post-menopausal women living in Mediterranean countries has been investigated in previous studies showing generally consistent results: in fact, higher adherence to the Mediterranean diet has been associated with better weight status and lower cardiovascular risk factors, such as blood lipids alteration and hyperuricemia [28-30]. In addition, an association between adherence to healthy dietary patterns such as the Mediterranean diet and improvement in at least one of the quality of life domains in older adults has been reported [31]. In line with such findings, a recent meta-analysis of seven prospective studies including a total of 11,738 elderly participants and 3874 deaths showed that increment in adherence to the Mediterranean diet was associated with $5 \%$ reduced risk of all-cause mortality [32]. Moreover, intervention studies suggested that counseling implementing adherence to a Mediterranean-type diet postmenopausal women living in non-Mediterranean countries (i.e., the United States or the United Kingdom) can favorably influence the diet quality and lipid profile [33] as well as improves microcirculatory vascular function and increases exercise tolerance, especially whether combined with regular moderate physical activity [34]. Interesting, higher adherence to the Mediterranean diet has been proven important also in secondary prevention of CVD, as a diet inspired by the Mediterranean pattern has been found associated with substantial reduction of total mortality of elderly patients after first myocardial infarction [35].

It may be argued that the Mediterranean diet may be associated with cultural (such as use of specific type of foods), lifestyle (such as eating and sleeping patterns) and even geographical factors (such as high sunlight exposure) characteristics of the 
Table 2

Odds ratios (ORs) e 95\% confidence intervals (CIs) for the association between high adherence to the Mediterranean diet (Q4 vs. Q1-Q3 quartile of the Mediterranean diet adherence score) and metabolic outcomes

\begin{tabular}{lccr}
\hline & \multicolumn{2}{c}{ High adherence to the Mediterranean diet $^{\text {OR }}$} & OR (95\% CI) \\
\cline { 2 - 4 } & Cases, $n(\%)$ & OR $(95 \% \text { CI })^{\mathrm{a}}$ & $0.52(0.31-0.86)$ \\
\hline Overweight (BMI > 25) & $296(56.3)$ & $0.77(0.59-1.00)$ & $0.38(0.18-0.78)$ \\
Obesity (BMI > 30) & $128(24.3)$ & $0.55(0.37-0.83)$ & $0.75(0.33-1.71)$ \\
Type-2 diabetes & $56(10.6)$ & $0.98(0.60-1.60)$ & $0.72(0.43-1.19)$ \\
Hypertension & $376(71.5)$ & $1.12(0.86-1.45)$ & $0.50(0.30-0.84)$ \\
Dyslipidemia & $216(41.1)$ & $0.75(0.53-1.07)$ & \\
\hline
\end{tabular}

${ }^{\mathrm{a}}$ Adjusted for total energy intake (kcal/d); ${ }^{\mathrm{b}}$ Adjusted for age, educational status, occupational status, marital status, smoking status, physical activity status, total energy intake ( $\mathrm{kcal} / \mathrm{d})$.

Table 3

Odds ratios (ORs) e 95\% confidence intervals (CIs) for the association between individual dietary components of the Mediterranean diet score and metabolic outcomes

\begin{tabular}{lcccccc}
\hline & \multicolumn{5}{c}{ OR $(95 \% \mathrm{CI})$} \\
\cline { 2 - 7 } & Criterion & Overweight & Obesity & Type-2 diabetes & Hypertension & Dyslipidemia \\
\hline Fruit $(150 \mathrm{~g})$ & $>2$ servings/d & $1.15(0.88-1.50)$ & $1.41(1.06-2.04)$ & $1.10(0.70-1.71)$ & $0.74(0.55-0.99)$ & $0.93(0.75-1.22)$ \\
Vegetables $(100 \mathrm{~g})$ & $>2.5$ servings/d & $1.12(0.82-1.53)$ & $0.84(0.58-1.20)$ & $1.63(0.93-2.88)$ & $0.89(0.64-1.26)$ & $1.32(0.95-1.82)$ \\
Legumes $(70 \mathrm{~g})$ & $>2$ servings/w & $0.90(0.70-1.17)$ & $0.72(0.54-0.97)$ & $1.21(0.76-1.95)$ & $1.02(0.76-1.36)$ & $0.68(0.52-0.89)$ \\
Dairy products $(180 \mathrm{~g})$ & $<1$ servings/d & $0.84(0.68-1-04)$ & $0.84(0.65-1.07)$ & $0.90(0.64-1.26)$ & $0.64(0.50-0.82)$ & $0.68(0.55-0.85)$ \\
Cereals $(130 \mathrm{~g})$ & $>1.5$ servings/d & $0.86(0.67-1.10)$ & $0.87(0.65-1.15)$ & $0.67(0.46-0.97)$ & $1.03(0.80-1.34)$ & $0.89(0.70-1.14)$ \\
Fish $(100 \mathrm{~g})$ & $>2.5$ servings/w & $1.27(0.94-1.73)$ & $1.35(0.90-2.20)$ & $1.68(0.93-3.03)$ & $1.32(0.94-1.84)$ & $0.97(0.71-1.33)$ \\
Alcohol $(12 \mathrm{~g})$ & $<1$ serving/d & $0.79(0.51-1.22)$ & $1.08(0.67-1.75)$ & $1.33(0.91-1.72)$ & $1.22(0.79-1.88)$ & $1.31(0.88-1.98)$ \\
Meat $(80 \mathrm{~g})$ & $<1$ serving/d & $0.90(0.67-1.22)$ & $0.74(0.54-1.03)$ & $1.30(0.80-2.12)$ & $1.16(0.84-1.59)$ & $1.15(0.85-1.55)$ \\
Olive oil & Regular use & $0.67(0.51-0.87)$ & $0.76(0.57-1.03)$ & $0.56(0.37-0.83)$ & $0.85(0.63-1.14)$ & $0.77(0.59-1.01)$ \\
\hline
\end{tabular}

Mediterranean area [36-39]. Previous studies conducted on the adult population of individuals living in Italy showed that adherence to Mediterranean diet is still high among older individuals, suggesting that dietary habits may have had played an important role on their health status over the lifespan [40-44]. Moreover, a number of reports conducted in population living outside the Mediterranean shores reported that individuals more adherent to a Mediterranean-type diet were less likely to suffer of metabolic disorders and cardiovascular risk factors [45-47]. However, an increasing trend over a "Westernization" of the diet has been observed also in the Mediterranean area [48-50]. In fact, paradoxically, findings on children and adolescents are far less consistent in studies within the Mediterranean area [51-54] and European investigations reported even higher impact on health in non-Mediterranean children [55]. However, a comprehensive evaluation of existing evidence from cohort studies showed a consistent inverse association between high adherence to the Mediterranean diet and metabolic syndrome occurrence [56]. Thus, biological mechanisms should be accounted for the potential effects of adherence to this dietary pattern and metabolic benefits.
There is no individual component of the diet that can be considered mainly responsible for the associations observed in this and several other studies. However, when our analysis was conducted to test the contribution of single food groups in the association with metabolic outcomes, we found significant results for nearly all foods investigated, despite on different outcomes, suggesting that the overall dietary quality of the Mediterranean dietary pattern may synergistically act toward human health at different levels. For instance, dietary carbohydrates characterizing the Mediterranean diet are provided by (whole) grains and legumes, which together with fruit and vegetable are rich sources of fiber and have been shown to be associated with lower risk of cardiometabolic outcomes [57-60]. Dietary sources of fats, such as fish, olive oil and nuts, provide mainly unsaturated fatty acids in substitution of saturated and trans-fatty acids, which have been associated with cardio-metabolic health [61, 62]. In addition, dairy products have been reported to be part of a healthy Mediterranean dietary pattern which, together with lower sodium consumption, may ameliorate cardiovascular risk [63, 64]. Moreover, several plant-foods commonly consumed, including those previously 
listed together with (red) wine, are rich in phytochemicals with antioxidant and anti-inflammatory properties, which may prevent or counteract the subclinical low-grade inflammation occurring with impaired body weight, metabolism, and generally considered the first pathogenic process occurring in cardio-metabolic disorders $[65,66]$. Among them, polyphenols have been among the most studied recently and have been associated with decreased risk of mortality (especially from CVD) as well as inversely associated with occurrence of type-2 diabetes, hypertension, and metabolic disorders [67, 68]. In addition, the moderate intake of alcohol has been hypothesized to be positive for cardiovascular health and it has been associated with lower incidence rates of metabolic syndrome features, including obesity, diabetes and hypertension [69].

Despite of interest, the results presented should be considered in light of some limitations that must be addressed. First, this was a cross-sectional study, thus it is descriptive of prevalence and merely descriptive rather than assessing causal relations. Second, methods used to collect nutrition data, such as FFQs, are affected by limitations, including recall bias, collinearity, may overestimate dietary intakes. Finally, despite the participant of this study were extracted from a representative sample as a whole, the sample of post-menopausal women analyzed in this study may not be representative.

In conclusions, adherence to the Mediterranean diet may be useful for a healthy aging and is associated with better metabolic status in women in post-menopause.

\section{Acknowledgment}

Authors disclose no conflict of interests. All authors approved the final version of the manuscript. This research was funded by the Department of Medical and Surgical Sciences and Advanced Technologies "G.F. Ingrassia," University of Catania, Italy (Piano Triennale di Sviluppo delle Attivita' di Ricerca Scientifica del Dipartimento 2016-18).

\section{References}

[1] Bechtold M, Palmer J, Valtos J, Iasiello C, Sowers J. Metabolic syndrome in the elderly. Curr Diab Rep. 2006;6(1):64-71.

[2] Collaboration NCDRF. Worldwide trends in body-mass index, underweight, overweight, and obesity from 1975 to 2016: A pooled analysis of 2416 population-based measurement studies in 128.9 million children, adolescents, and adults. Lancet. 2017;390(10113):2627-42.

[3] Collaborators GBDRF. Global, regional, and national comparative risk assessment of 84 behavioural, environmental and occupational, and metabolic risks or clusters of risks for 195 countries and territories, 1990-2017: A systematic analysis for the Global Burden of Disease Study 2017. Lancet. 2018;392(10159):1923-94.

[4] Collaborators GBDCoD. Global, regional, and national age-sex-specific mortality for 282 causes of death in 195 countries and territories, 1980-2017: A systematic analysis for the Global Burden of Disease Study 2017. Lancet. 2018;392(10159):1736-88.

[5] DALYs GBD, Collaborators H. Global, regional, and national disability-adjusted life-years (DALYs) for 359 diseases and injuries and healthy life expectancy (HALE) for 195 countries and territories, 1990-2017: A systematic analysis for the Global Burden of Disease Study 2017. Lancet. 2018;392(10159):1859-922.

[6] Howard BV, Kuller L, Langer R, Manson JE, Allen C, Assaf A, Cochrane BB, Larson JC, Lasser N, Rainford M, Van Horn L, Stefanick ML, Trevisan M, Women's Health I. Risk of cardiovascular disease by hysterectomy status, with and without oophorectomy: The Women's Health Initiative Observational Study. Circulation. 2005;111(12):1462-70.

[7] Bertone-Johnson ER, Whitcomb BW, Rich-Edwards JW, Hankinson SE, Manson JE. Premenstrual Syndrome and Subsequent Risk of Hypertension in a Prospective Study. Am J Epidemiol. 2015;182(12):1000-9.

[8] Cirillo PM, Cohn BA. Pregnancy complications and cardiovascular disease death: 50-year follow-up of the Child Health and Development Studies pregnancy cohort. Circulation. 2015;132(13):1234-42.

[9] Roach RE, Helmerhorst FM, Lijfering WM, Stijnen T, Algra A, Dekkers OM. Combined oral contraceptives: The risk of myocardial infarction and ischemic stroke. Cochrane Database Syst Rev. 2015(8):CD011054.

[10] Yang XP, Reckelhoff JF. Estrogen, hormonal replacement therapy and cardiovascular disease. Curr Opin Nephrol Hypertens. 2011;20(2):133-8.

[11] Lobo RA, Davis SR, De Villiers TJ, Gompel A, Henderson VW, Hodis HN, Lumsden MA, Mack WJ, Shapiro S, Baber RJ. Prevention of diseases after menopause. Climacteric. 2014;17(5):540-56

[12] Mastorakos G, Valsamakis G, Paltoglou G, Creatsas G. Management of obesity in menopause: Diet, exercise, pharmacotherapy and bariatric surgery. Maturitas. 2010;65(3):219-24.

[13] Grosso G, Marventano S, Yang J, Micek A, Pajak A, Scalfi L, Galvano F, Kales SN. A comprehensive meta-analysis on evidence of Mediterranean diet and cardiovascular disease: Are individual components equal? Crit Rev Food Sci Nutr. 2017;57(15):3218-32.

[14] Liyanage T, Ninomiya T, Wang A, Neal B, Jun M, Wong MG, Jardine M, Hillis GS, Perkovic V. Effects of the Mediterranean Diet on Cardiovascular OutcomesA Systematic Review and Meta-Analysis. PLoS One. 2016;11(8):e0159252.

[15] D'Alessandro A, De Pergola G. The Mediterranean Diet: Its definition and evaluation of a priori dietary indexes in primary cardiovascular prevention. Int J Food Sci Nutr. 2018;69(6):647-59. 
[16] Davis C, Bryan J, Hodgson J, Murphy K. Definition of the Mediterranean Diet; a Literature Review. Nutrients. 2015;7(11):9139-53

[17] Garcia M, Bihuniak JD, Shook J, Kenny A, Kerstetter J, Huedo-Medina TB. The Effect of the Traditional Mediterranean-Style Diet on Metabolic Risk Factors: A Meta-Analysis. Nutrients. 2016;8(3):168.

[18] Grosso G, Mistretta A, Frigiola A, Gruttadauria S, Biondi A, Basile F, Vitaglione P, D’Orazio N, Galvano F. Mediterranean diet and cardiovascular risk factors: A systematic review. Crit Rev Food Sci Nutr. 2014;54(5):593-610.

[19] Godos J, Federico A, Dallio M, Scazzina F. Mediterranean diet and nonalcoholic fatty liver disease: Molecular mechanisms of protection. Int J Food Sci Nutr. 2017;68(1):18-27.

[20] Grosso G, Marventano S, D’Urso M, Mistretta A, Galvano F. The Mediterranean healthy eating, ageing, and lifestyle (MEAL) study: Rationale and study design. International Journal of Food Sciences and Nutrition. 2016;68(5):1-10.

[21] Marventano S, Mistretta A, Platania A, Galvano F, Grosso G. Reliability and relative validity of a food frequency questionnaire for Italian adults living in Sicily, Southern Italy. International Journal of Food Sciences and Nutrition. 2016; 67(7):1-8

[22] Buscemi S, Rosafio G, Vasto S, Massenti FM, Grosso G, Galvano F, Rini N, Barile AM, Maniaci V, Cosentino L, Verga $\mathrm{S}$. Validation of a food frequency questionnaire for use in Italian adults living in Sicily. International Journal of Food Sciences and Nutrition. 2015;66(4):426-38.

[23] Sofi F, Dinu M, Pagliai G, Marcucci R, Casini A. Validation of a literature-based adherence score to Mediterranean diet: The MEDI-LITE score. Int J Food Sci Nutr. 2017;68(6):757-62.

[24] Mistretta A, Marventano S, Platania A, Godos J, Galvano F, Grosso G. Metabolic profile of the Mediterranean healthy Eating, Lifestyle and Aging (MEAL) study cohort. Mediterranean Journal of Nutrition and Metabolism. 2017;10(2):131-40.

[25] Platania A, Zappala G, Mirabella MU, Gullo C, Mellini G, Beneventano G, Maugeri G, Marranzano M. Association between Mediterranean diet adherence and dyslipidaemia in a cohort of adults living in the Mediterranean area. Int J Food Sci Nutr. 2018;69(5):608-18.

[26] Zappala G, Buscemi S, Mule S, La Verde M, D’Urso M, Corleo D, Marranzano M. High adherence to Mediterranean diet, but not individual foods or nutrients, is associated with lower likelihood of being obese in a Mediterranean cohort. Eat Weight Disord. 2018;23(5):605-14.

[27] Grosso G, Pajak A, Mistretta A, Marventano S, Raciti T, Buscemi S, Drago F, Scalfi L, Galvano F. Protective role of the Mediterranean diet on several cardiovascular risk factors: Evidence from Sicily, southern Italy. Nutr Metab Cardiovasc Dis. 2014;24(4):370-7.

[28] Panagiotakos DB, Polystipioti A, Papairakleous N, Polychronopoulos E. Long-term adoption of a Mediterranean diet is associated with a better health status in elderly people; a cross-sectional survey in Cyprus. Asia Pac J Clin Nutr. 2007;16(2):331-7.

[29] Tyrovolas S, Bountziouka V, Papairakleous N, Zeimbekis A, Anastassiou F, Gotsis E, Metallinos G, Polychronopoulos E, Lionis C, Panagiotakos D. Adherence to the Mediterranean diet is associated with lower prevalence of obesity among elderly people living in Mediterranean islands: The MEDIS study. Int J Food Sci Nutr. 2009;60(Suppl 6):137-50.
[30] Chrysohoou C, Skoumas J, Pitsavos C, Masoura C, Siasos G, Galiatsatos N, Psaltopoulou T, Mylonakis C, Margazas A, Kyvelou S, Mamatas S, Panagiotakos D, Stefanadis C. Long-term adherence to the Mediterranean diet reduces the prevalence of hyperuricaemia in elderly individuals, without known cardiovascular disease: The Ikaria study. Maturitas. 2011;70(1):58-64.

[31] Govindaraju T, Sahle BW, McCaffrey TA, McNeil JJ, Owen AJ. Dietary Patterns and Quality of Life in Older Adults: A Systematic Review. Nutrients. 2018;10(8)

[32] Bonaccio M, Di Castelnuovo A, Costanzo S, Gialluisi A, Persichillo M, Cerletti C, Donati MB, de Gaetano G, Iacoviello L. Mediterranean diet and mortality in the elderly: A prospective cohort study and a meta-analysis. The British Journal of Nutrition. 2018;120(8):841-54.

[33] Bihuniak JD, Ramos A, Huedo-Medina T, Hutchins-Wiese H, Kerstetter JE, Kenny AM. Adherence to a MediterraneanStyle Diet and Its Influence on Cardiovascular Risk Factors in Postmenopausal Women. J Acad Nutr Diet. 2016;116(11):1767-75.

[34] Alkhatib A, Klonizakis M. Effects of exercise training and Mediterranean diet on vascular risk reduction in post-menopausal women. Clin Hemorheol Microcirc. 2014;57(1):33-47.

[35] Trichopoulou A, Bamia C, Norat T, Overvad K, Schmidt EB, Tjonneland A, Halkjaer J, Clavel-Chapelon F, Vercambre MN, Boutron-Ruault MC, Linseisen J, Rohrmann S, Boeing $\mathrm{H}$, Weikert $\mathrm{C}$, Benetou V, Psaltopoulou T, Orfanos P, Boffetta P, Masala G, Pala V, Panico S, Tumino R, Sacerdote C, Bueno-de-Mesquita HB, Ocke MC, Peeters PH, Van der Schouw YT, Gonzalez C, Sanchez MJ, Chirlaque MD, Moreno C, Larranaga N, Van Guelpen B, Jansson JH, Bingham S, Khaw KT, Spencer EA, Key T, Riboli E, Trichopoulos D. Modified Mediterranean diet and survival after myocardial infarction: The EPIC-Elderly study. Eur J Epidemiol. 2007;22(12):871-81.

[36] Mocciaro G, Ziauddeen N, Godos J, Marranzano M, Chan MY, Ray S. Does a Mediterranean-type dietary pattern exert a cardio-protective effect outside the Mediterranean region? A review of current evidence. Int J Food Sci Nutr. 2018;69(5):524-35.

[37] Alexandratos N. The Mediterranean diet in a world context. Public Health Nutrition. 2006;9(1A):111-7.

[38] da Silva R, Bach-Faig A, Raido Quintana B, Buckland G, Vaz de Almeida MD, Serra-Majem L. Worldwide variation of adherence to the Mediterranean diet, in 1961-1965 and 2000-2003. Public Health Nutrition. 2009;12(9A):1676-84.

[39] Moreno-Altamirano L, Hernández-Montoya D, Soto-Estrada G, García-García J, Silberman M, Capraro S, Panico S. Changes in Mediterranean dietary patterns in Italy from 1961 to 2011. Mediterranean Journal of Nutrition and Metabolism. 2016;9(3):171-81.

[40] Bonaccio M, Bes-Rastrollo M, de Gaetano G, Iacoviello L. Challenges to the Mediterranean diet at a time of economic crisis. Nutr Metab Cardiovasc Dis. 2016;26(12):1057-63.

[41] Pelucchi C, Galeone C, Negri E, La Vecchia C. Trends in adherence to the Mediterranean diet in an Italian population between 1991 and 2006. Eur J Clin Nutr. 2010;64(10): 1052-6.

[42] Grosso G, Marventano S, Giorgianni G, Raciti T, Galvano F, Mistretta A. Mediterranean diet adherence rates in Sicily, southern Italy. Public Health Nutr. 2014;17(9):2001-9. 
[43] Vasto S, Buscemi S, Barera A, Di Carlo M, Accardi G, Caruso C. Mediterranean diet and healthy ageing: A Sicilian perspective. Gerontology. 2014;60(6):508-18.

[44] Marventano S, Godos J, Platania A, Galvano F, Mistretta A, Grosso G. Mediterranean diet adherence in the Mediterranean healthy eating, aging and lifestyle (MEAL) study cohort. Int J Food Sci Nutr. 2018;69(1):100-7.

[45] Carter SJ, Roberts MB, Salter J, Eaton CB. Relationship between Mediterranean Diet Score and atherothrombotic risk: Findings from the Third National Health and Nutrition Examination Survey (NHANES III), 1988-1994. Atherosclerosis. 2010;210(2):630-6.

[46] Yang J, Farioli A, Korre M, Kales SN. Modified Mediterranean diet score and cardiovascular risk in a North American working population. PLoS One. 2014;9(2):e87539.

[47] Grosso G, Stepaniak U, Micek A, Topor-Madry R, Stefler D, Szafraniec K, Bobak M, Pajak A. A Mediterranean-type diet is associated with better metabolic profile in urban Polish adults: Results from the HAPIEE study. Metabolism. 2015;64(6):738-46.

[48] Grosso G, Galvano F. Mediterranean diet adherence in children and adolescents in southern European countries. NFS Journal. 2016;3:13-9.

[49] Balanza R, Garcia-Lorda P, Perez-Rodrigo C, Aranceta J, Bonet MB, Salas-Salvado J. Trends in food availability determined by the Food and Agriculture Organization's food balance sheets in Mediterranean Europe in comparison with other European areas. Public Health Nutrition. 2007;10(2):168-76.

[50] Vareiro D, Bach-Faig A, Raido Quintana B, Bertomeu I, Buckland G, Vaz de Almeida MD, Serra-Majem L. Availability of Mediterranean and non-Mediterranean foods during the last four decades: Comparison of several geographical areas. Public Health Nutrition. 2009;12(9A):1667-75.

[51] Santomauro F, Lorini C, Tanini T, Indiani L, Lastrucci V, Comodo N, Bonaccorsi G. Adherence to Mediterranean diet in a sample of Tuscan adolescents. Nutrition. 2014;30(1112):1379-83.

[52] Velazquez-Lopez L, Santiago-Diaz G, Nava-Hernandez J, Munoz-Torres AV, Medina-Bravo P, Torres-Tamayo M. Mediterranean-style diet reduces metabolic syndrome components in obese children and adolescents with obesity. BMC Pediatr. 2014; 14:175.

[53] Grosso G, Marventano S, Buscemi S, Scuderi A, Matalone M, Platania A, Giorgianni G, Rametta S, Nolfo F, Galvano F, Mistretta A. Factors associated with adherence to the Mediterranean diet among adolescents living in Sicily, Southern Italy. Nutrients. 2013;5(12):4908-23.

[54] Mistretta A, Marventano S, Antoci M, Cagnetti A, Giogianni G, Nolfo F, Rametta S, Pecora G, Marranzano M. Mediterranean diet adherence and body composition among Southern Italian adolescents. Obes Res Clin Pract. 2016.

[55] Tognon G, Hebestreit A, Lanfer A, Moreno LA, Pala V, Siani A, Tornaritis M, De Henauw S, Veidebaum T, Molnar D, Ahrens W, Lissner L. Mediterranean diet, overweight and body composition in children from eight European countries: Cross-sectional and prospective results from the IDEFICS study. Nutrition, metabolism, and cardiovascular diseases: NMCD. 2014;24(2):205-13.

[56] Godos J, Zappala G, Bernardini S, Giambini I, Bes-Rastrollo M, Martinez-Gonzalez M. Adherence to the Mediterranean diet is inversely associated with metabolic syndrome occur- rence: A meta-analysis of observational studies. Int J Food Sci Nutr. 2017;68(2):138-48.

[57] Boeing H, Bechthold A, Bub A, Ellinger S, Haller D, Kroke A, Leschik-Bonnet E, Muller MJ, Oberritter H, Schulze M, Stehle P, Watzl B. Critical review: Vegetables and fruit in the prevention of chronic diseases. European Journal of Nutrition. 2012;51(6):637-63.

[58] Schwingshackl L, Hoffmann G, Kalle-Uhlmann T, Arregui M, Buijsse B, Boeing H. Fruit and Vegetable Consumption and Changes in Anthropometric Variables in Adult Populations: A Systematic Review and MetaAnalysis of Prospective Cohort Studies. PLoS One. 2015;10(10):e0140846.

[59] Rebello CJ, Greenway FL, Finley JW. A review of the nutritional value of legumes and their effects on obesity and its related co-morbidities. Obesity reviews: An official journal of the International Association for the Study of Obesity. 2014;15(5):392-407.

[60] Marventano S, Izquierdo Pulido M, Sanchez-Gonzalez C, Godos J, Speciani A, Galvano F, Grosso G. Legume consumption and CVD risk: A systematic review and metaanalysis. Public Health Nutr. 2017;20(2):245-54.

[61] Marventano S, Kolacz P, Castellano S, Galvano F, Buscemi S, Mistretta A, Grosso G. A review of recent evidence in human studies of n-3 and n-6 PUFA intake on cardiovascular disease, cancer, and depressive disorders: Does the ratio really matter? Int J Food Sci Nutr. 2015;66(6):611-22.

[62] Grosso G, Estruch R. Nut consumption and age-related disease. Maturitas. 2016;84:11-6.

[63] La Verde M, Mule S, Zappala G, Privitera G, Maugeri G, Pecora F, Marranzano M. Higher adherence to the Mediterranean diet is inversely associated with having hypertension: Is low salt intake a mediating factor? Int J Food Sci Nutr. 2018;69(2):235-44.

[64] De Pergola G, D'Alessandro A. Influence of Mediterranean Diet on Blood Pressure. Nutrients. 2018;10(11).

[65] Castro-Barquero S, Lamuela-Raventos RM, Domenech M, Estruch R. Relationship between Mediterranean Dietary Polyphenol Intake and Obesity. Nutrients. 2018;10(10).

[66] Marranzano M, Ray S, Godos J, Galvano F. Association between dietary flavonoids intake and obesity in a cohort of adults living in the Mediterranean area. Int J Food Sci Nutr. 2018;69(8):1020-9.

[67] Grosso G, Micek A, Godos J, Pajak A, Sciacca S, Galvano F, Giovannucci EL. Dietary Flavonoid and Lignan Intake and Mortality in Prospective Cohort Studies: Systematic Review and Dose-Response Meta-Analysis. Am J Epidemiol. 2017;185(12):1304-16.

[68] Rienks J, Barbaresko J, Nothlings U. Association of Polyphenol Biomarkers with Cardiovascular Disease and Mortality Risk: A Systematic Review and Meta-Analysis of Observational Studies. Nutrients. 2017;9(4).

[69] Tresserra-Rimbau A, Medina-Remon A, Lamuela-Raventos RM, Bullo M, Salas-Salvado J, Corella D, Fito M, Gea A, Gomez-Gracia E, Lapetra J, Aros F, Fiol M, Ros E, SerraMajem L, Pinto X, Munoz MA, Estruch R, Investigators PS. Moderate red wine consumption is associated with a lower prevalence of the metabolic syndrome in the PREDIMED population. The British Journal of Nutrition. 2015;113(Suppl 2):S121-30. 